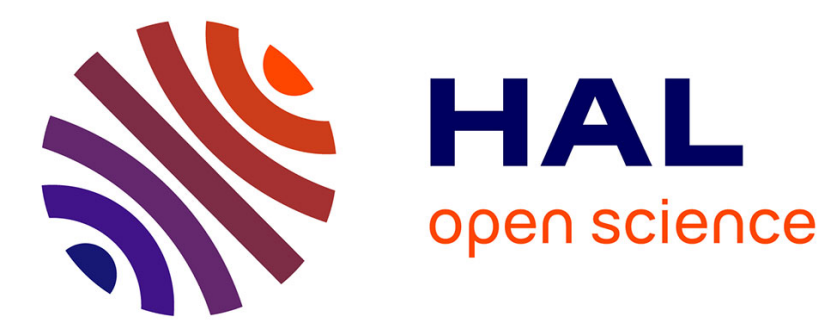

\title{
Une leishmaniose cutanée réfractaire : intérêt de la miltéfosine
}

\author{
C. Guerveno, K. Delavigne, A. Berry, G. Martin-Blondel, P. Delobel
}

\section{To cite this version:}

C. Guerveno, K. Delavigne, A. Berry, G. Martin-Blondel, P. Delobel. Une leishmaniose cutanée réfractaire: intérêt de la miltéfosine. Médecine et Maladies Infectieuses, 2019, 49 (4), pp.281 - 283. 10.1016/j.medmal.2018.11.009 . hal-03486839

\section{HAL Id: hal-03486839 \\ https://hal.science/hal-03486839}

Submitted on 20 Dec 2021

HAL is a multi-disciplinary open access archive for the deposit and dissemination of scientific research documents, whether they are published or not. The documents may come from teaching and research institutions in France or abroad, or from public or private research centers.
L'archive ouverte pluridisciplinaire HAL, est destinée au dépôt et à la diffusion de documents scientifiques de niveau recherche, publiés ou non, émanant des établissements d'enseignement et de recherche français ou étrangers, des laboratoires publics ou privés.

\section{다)(1) $(5$}

Distributed under a Creative Commons Attribution - NonCommerciall 4.0 International 


\section{Une leishmaniose cutanée réfractaire : intérêt de la miltéfosine Refractory cutaneous leishmaniasis : benefit of miltefosine}

$$
\text { C Guerveno }^{1} \text {, K Delavigne }{ }^{2} \text {, A Berry }{ }^{3,4,5} \text {, G Martin-Blondel }{ }^{1,4,5} \text {, P Delobel }{ }^{1,4,5}
$$

${ }^{1} \mathrm{CHU}$ de Toulouse, Service des Maladies Infectieuses et Tropicales, Toulouse, F-31300 France

${ }^{2} \mathrm{CHU}$ de Toulouse, Service de Médecine Interne, Toulouse, F-31300 France

${ }^{3} \mathrm{CHU}$ de Toulouse, Laboratoire de Parasitologie, Toulouse, F-31300 France

${ }^{4}$ INSERM, UMR1043, Toulouse, F-31300 France

${ }^{5}$ Université Toulouse III Paul Sabatier, Toulouse, F-31300 France

Les auteurs déclarent ne pas avoir de conflit d'intérêt pour cet article.

Correspondance : Pr Pierre Delobel, Service des Maladies Infectieuses et Tropicales, CHU de Toulouse, F-31300 France. Tel: 05617775 08; fax: 05617721 38; e-mail: delobel.p@chutoulouse.fr

Mots clés: leishmaniose cutanée, Leishmania tropica, miltéfosine

Keywords: dermal leishmaniasis ; Leishmania tropica, miltefosine 


\section{Introduction}

Différentes espèces de Leishmania sont responsables des leishmanioses dites de l'Ancien Monde et du Nouveau Monde (Amériques). Les leishmanioses de l'Ancien Monde sont dues à Leishmania infantum et $L$. donovani pour les formes viscérales, et à $L$. tropica, $L$. major, et L. aethiopica, pour les formes cutanées. La leishmaniose cutanée à L. tropica s'observe essentiellement dans le bassin méditerranéen et au Moyen-Orient [1]. Elle est transmise par la piqûre d'un phlébotome femelle et l'homme est le réservoir quasi-exclusif de $L$. tropica, comme pour L. donovani, à la différence de la plupart des autres espèces de Leishmania qui sont des anthropozoonoses. Les lésions cutanées sont habituellement d'évolution chronique. Le traitement des leishmanioses cutanées de l'Ancien Monde repose sur l'abstention thérapeutique ou des traitements locaux (cryothérapie, injections intralésionnelles de dérivés de l'antimoine, applications de paromomycine) dans les formes localisées de petite taille, ou sur des traitements systémiques pour les lésions plus étendues [1, 2]. Les dérivés de l'antimoine constituent le traitement systémique de référence mais leur utilisation est limitée par leur toxicité. L'amphotéricine B, notamment dans sa forme liposomale, la pentamidine, ou le fluconazole, sont également proposés comme alternatives aux dérivés de l'antimoine $[1,2]$.

Nous décrivons un cas de leishmaniose cutanée à $L$. tropica en échec de tous les traitements systémiques usuels chez un patient immunodéprimé, mais d'évolution rapidement favorable sous miltéfosine, traitement habituellement réservé aux leishmanioses viscérales réfractaires et dont l'efficacité dans les leishmanioses cutanées de l'Ancien Monde est encore mal établie. 


\section{Observation}

Un homme de 72 ans a consulté pour la prise en charge d'une lésion cutanée subaiguë. Dans les semaines suivant un voyage en Syrie, une lésion cutanée unique était apparue en regard de la scapula gauche, de taille croissante jusqu'à $5 \mathrm{~cm}$ de diamètre, infiltrée et partiellement ulcérée (Figure 1). Des leishmanies amastigotes ont été observées à l'examen anatomopathologique de la biopsie cutanée, et le diagnostic a été confirmé par PCR. Le séquençage a permis d'identifier L. tropica.

Le bilan biologique a mis en évidence par ailleurs une lymphopénie à $0,4 \mathrm{G} / \mathrm{L}$ sans autres anomalies de I'hémogramme, associée à une hypo- $\gamma$-globulinémie à 3,2 $\mathrm{g} / \mathrm{L}$ avec un déficit en IgG, IgA et IgM. II n'a pas été observé pas de composant monoclonal par l'immunoélectrophorèse des protides sanguins et urinaires. Des lymphocytes T CD4 $4^{+}$à $129 / \mu \mathrm{L}(33 \%)$, des lymphocytes $\mathrm{T} \mathrm{CD}^{+}$à $185 / \mu \mathrm{L}(33 \%)$, et des lymphocytes B à 34/ $\mu \mathrm{L}(6 \%)$ avec un déficit sélectif des lymphocytes B mémoires (9,9\%) ont été mis en évidence par l'immunophénotypage des lymphocytes sanguins. La sérologie VIH était négative. La recherche d'hémopathie acquise était négative (tomodensitométrie thoraco-abdominopelvienne, myélogramme, et biopsie ostéo-médullaire normaux). Le diagnostic de Déficit Immunitaire Commun Variable associé à une lymphopénie $T \mathrm{CD}^{+}$a été retenu. Le patient n'avait jamais eu auparavant d'expression clinique de ce déficit immunitaire biologique, notamment pas d'antécédents d'infections ORL ou respiratoires.

Devant la taille de la lésion cutanée de Leishmaniose, l'indication d'un traitement systémique a été portée : antimoniate de méglumine, $3 \mathrm{~g} / \mathrm{jour}$ soit $810 \mathrm{mg} / \mathrm{j}$ d'antimoine, par voie intramusculaire pendant 21 jours. Devant l'absence d'amélioration, il a alors été traité par pentamidine, en injection unique de $7 \mathrm{mg} / \mathrm{kg}$ par voie intramusculaire, selon le 
schéma utilisé pour L. guyanensis [3], avec une efficacité partielle, injection renouvelée 5 semaines plus tard, mais qui n’a pas amené à la guérison. Devant la persistance de la lésion à 3 mois de la deuxième injection de pentamidine, 10 perfusions d'amphotéricine $B$ liposomale à la posologie de $4 \mathrm{mg} / \mathrm{kg} / \mathrm{jour}$ ont été administrées, soit $40 \mathrm{mg} / \mathrm{kg}$ en dose cumulée. La lésion a régressé partiellement mais toujours sans parvenir à une guérison complète. Un traitement additionnel par fluconazole, $400 \mathrm{mg} / \mathrm{jour}$ par voie orale pendant 3 mois a été prescrit, sans permettre d'amélioration significative.

Devant l'aspect réfractaire de la leishmaniose cutanée après 18 mois de traitements successifs par dérivés de l'antimoine, pentamidine, amphotéricine $B$, et fluconazole, il a été proposé au patient un traitement par miltéfosine après obtention d'une Autorisation Temporaire d'Utilisation nominative par l'Agence Nationale de Sécurité du Médicaments et des produits de santé (ANSM). La posologie débutée à $150 \mathrm{mg} / \mathrm{jour}$ en 3 prises par voie orale a été diminuée à $100 \mathrm{mg} / \mathrm{jour}$ en 2 prises devant des troubles digestifs. Une guérison complète de la lésion a été obtenue après 8 semaines de traitement (Figure 2), sans récidive ultérieure.

\section{Discussion}

La stratégie de prise en charge thérapeutique des leishmanioses cutanées repose sur les caractéristiques des lésions et de l'espèce incriminée et le terrain immunocompétent ou non du sujet infecté $[1,2]$. En cas de lésions de taille supérieure à $30 \mathrm{~mm}$, de lésions multiples, ou d'espèces à risque de localisations secondaires muqueuses pour certaines leishmanioses du Nouveau Monde, le traitement local est insuffisant et un traitement systémique est proposé. Concernant les leishmanioses à L. tropica, les dérivés de l'antimoine par voie 
parentérale restent à ce jour le traitement de référence recommandé [1, 2]. Les taux de guérison rapporté restent néanmoins relativement faibles (41 à 55\%) avec les dérivés de l'antimoine pour L. tropica [2].

Dans notre cas, la leishmaniose était réfractaire aux différents traitements reçus, échecs probablement favorisés par l'immunodépression sous-jacente lié à la lymphopénie T CD4 associée au DICV. Par analogie avec le traitement des leishmanioses viscérales, un traitement par miltéfosine a donc été proposé et s'est révélé efficace de manière spectaculaire en 2 mois après plus de 18 mois de traitements successifs inefficaces.

La miltéfosine est un alkyl-lysophospholipide. II s'agit d'un traitement par voie orale ayant des effets secondaires essentiellement digestifs avec des vomissements et des douleurs abdominales, alors que les dérivés de l'antimoine sont responsables de toxicité systémique potentiellement sévère au niveau cardiaque, rénale ou hématologique. Néanmoins, l'utilisation de la miltéfosine est limitée par son risque tératogène et son coût élevé. Son efficacité dans les leishmanioses viscérales, notamment $L$. donovani, est établie même s'il existe des résistances croissantes dans le sous-continent indien. En revanche son utilisation dans les leishmanioses cutanées n'est pas mentionnée dans les recommandations officielles, exceptées pour les trois espèces du sous-genre Viannia, L. (V.) braziliensis, L. (V.) panamensis et L. (V.) guyanensis où la miltéfosine est un traitement validé par la Food and Drug Administration des Etats-Unis d'Amerique [1]. Cette recommandation repose sur deux études randomisées ayant évalué l'efficacité de la miltéfosine vs. dérivés de l'antimoine dans les leishmanioses cutanées du Nouveau Monde avec des taux de succès de $75 \%$ pour $L$. braziliensis [4], et $71.4 \%$ pour L. guyanensis [5]. 
Concernant les leishmanioses cutanées de l'Ancien Monde, une étude in vitro a montré l'action cytotoxique de la miltéfosine sur L. tropica et $L$. major avec une sensibilité légèrement supérieure pour L. tropica vs. L. major [6]. En 2008, l'efficacité clinique de la miltéfosine est décrite sur des leishmanioses cutanées chez des soldats canadiens au retour d’Afghanistan après échec du fluconazole : 2 cas étaient prouvés à $L$. tropica et le traitement reçu était de $150 \mathrm{mg} / \mathrm{j}$ pendant 28 jours [7]. Deux autres cas de militaires présentant une leishmaniose cutanée à $L$. braziliensis et $L$. infantum sont également décrits avec une réponse à la miltéfosine après 28 jours de traitement à $150 \mathrm{mg} / \mathrm{j}$ [8]. Un seul essai randomisé a été réalisé concernant les leishmanioses cutanées de l'Ancien Monde liées à $L$. major en Iran, comparant miltéfosine et antimoniate de meglumine avec une efficacité équivalente du traitement de $81,3 \%$ pour la miltéfosine vs. $80,6 \%$ pour l'antimoniate de meglumine [9]. Dans cette étude, sur 32 patients traités par miltéfosine, 4 ont interrompu le traitement précocement en raison d'effets secondaires digestifs, et sur 28 patients ayant poursuivi le traitement, 1 échec et 1 rechute étaient notés. Dans une revue de la littérature publiée en 2016, 24 cas de leishmanioses cutanées de l'Ancien Monde traités par miltéfosine sont recensés, mais seuls 3 cas concernent $L$. tropica, espèce en cause chez notre patient [10]. L'ensemble des cas ont montré une guérison des lésions sans effets secondaires graves, avec seulement 2 rechutes dans un contexte d'immunodépression.

Le cas que nous rapportons est donc à notre connaissance le $4^{\text {ème }}$ cas dans la littérature de traitement d'une leishmaniose de l'Ancien Monde à L. tropica par la miltéfosine, chez un patient immunodéprimé en échec de tous les traitements systémiques usuels.

\section{Conclusion}


La miltéfosine est une thérapeutique à envisager en cas de leishmaniose cutanée réfractaire au traitement usuel. Des études randomisées sont nécessaires pour confirmer son efficacité notamment dans les leishmanioses de l'Ancien Monde où il existe encore peu de données actuellement.

\section{Remerciements}

P.D. et K.D. ont traité le patient; A.B. a fait le diagnostic parasitologique ; G.M. a relu et corrigé le manuscrit ; C.G. et P.D. ont rédigé le manuscrit. 


\section{Références}

[1] Aronson N, Herwaldt BL, Libman M, Pearson R, Lopez-Velez R, Weina P, et al. Diagnosis and Treatment of Leishmaniasis: Clinical Practice Guidelines by the Infectious Diseases Society of America (IDSA) and the American Society of Tropical Medicine and Hygiene (ASTMH). Clin Infect Dis. 2016;63:e202-e64.

[2] Blum J, Buffet P, Visser L, Harms G, Bailey MS, Caumes E, et al. LeishMan recommendations for treatment of cutaneous and mucosal leishmaniasis in travelers, 2014. J Travel Med. 2014;21:116-29.

[3] Roussel M, Nacher M, Fremont G, Rotureau B, Clyti E, Sainte-Marie D, et al. Comparison between one and two injections of pentamidine isethionate, at $7 \mathrm{mg} / \mathrm{kg}$ in each injection, in the treatment of cutaneous leishmaniasis in French Guiana. Ann Trop Med Parasitol. 2006;100:307-14.

[4] Machado PR, Ampuero J, Guimaraes LH, Villasboas L, Rocha AT, Schriefer A, et al. Miltefosine in the treatment of cutaneous leishmaniasis caused by Leishmania braziliensis in Brazil: a randomized and controlled trial. PLoS Negl Trop Dis. 2010;4:e912.

[5] Chrusciak-Talhari A, Dietze R, Chrusciak Talhari C, da Silva RM, Gadelha Yamashita EP, de Oliveira Penna G, et al. Randomized controlled clinical trial to access efficacy and safety of miltefosine in the treatment of cutaneous leishmaniasis Caused by Leishmania (Viannia) guyanensis in Manaus, Brazil. Am J Trop Med Hyg. 2011;84:255-60.

[6] Khademvatan S, Gharavi MJ, Rahim F, Saki J. Miltefosine-induced apoptotic cell death on Leishmania major and L. tropica strains. Korean J Parasitol. 2011;49:17-23.

[7] Keynan Y, Larios OE, Wiseman MC, Plourde M, Ouellette M, Rubinstein E. Use of oral miltefosine for cutaneous leishmaniasis in Canadian soldiers returning from Afghanistan. Can $\mathrm{J}$ Infect Dis Med Microbiol. 2008;19:394-6.

[8] van der Snoek EM, Couwenberg SM, Stijnis C, Kortbeek LM, Schadd EM. Two cases of cutaneous leishmaniasis in Dutch military personnel treated with oral miltefosine. J R Army Med Corps. 2017;163:68-70.

[9] Mohebali M, Fotouhi A, Hooshmand B, Zarei Z, Akhoundi B, Rahnema A, et al. Comparison of miltefosine and meglumine antimoniate for the treatment of zoonotic cutaneous leishmaniasis (ZCL) by a randomized clinical trial in Iran. Acta Trop. 2007;103:3340.

[10] Mosimann V, Blazek C, Grob H, Chaney M, Neumayr A, Blum J. Miltefosine for Mucosal and Complicated Cutaneous Old World Leishmaniasis: A Case Series and Review of the Literature. Open Forum Infect Dis. 2016;3:ofw008. 
Figure 1. Lésion initiale de leishmaniose cutanée chez le patient au diagnostic. Lésion infiltrée et ulcérée, de localisation scapulaire gauche.

Figure 1. Initial cutaneous lesion at the time of diagnosis. Ulcerative and infiltrative lesion in the area of left scapula.

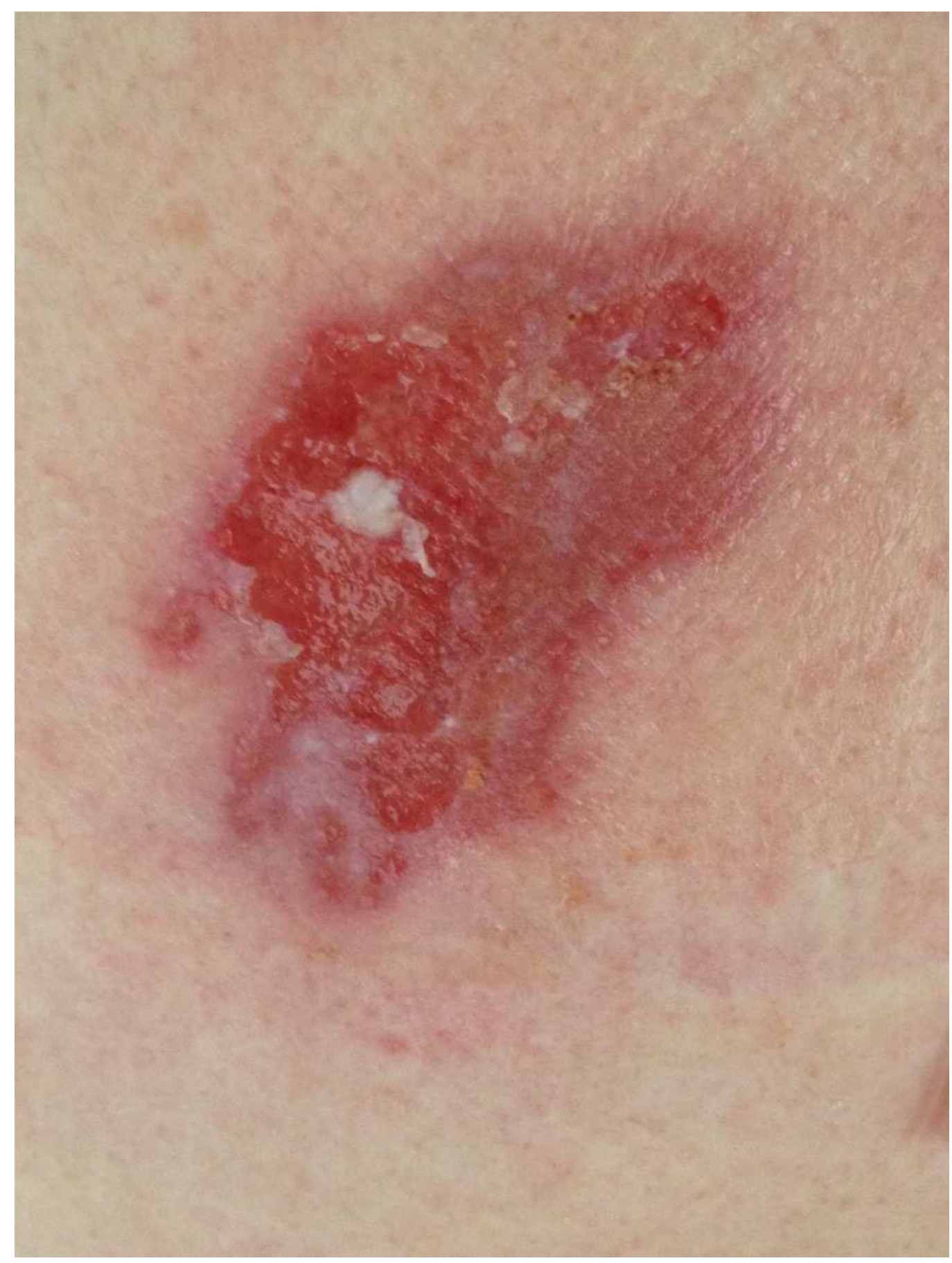


Figure 2. Guérison complète de la lésion de leishmaniose cutanée après traitement par miltéfosine.

Figure 2. Complete healing of the lesion after miltefosine treatment.



